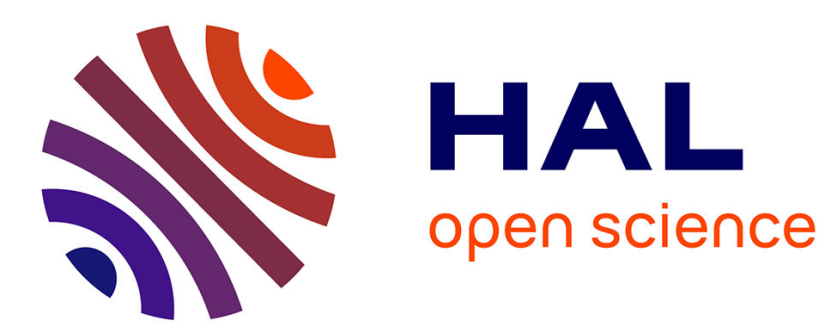

\title{
A three-dimensional constitutive model for the large stretch behavior of rubber elastic materials
}

Ellen M. Arruda, Mary C. Boyce

\section{To cite this version:}

Ellen M. Arruda, Mary C. Boyce. A three-dimensional constitutive model for the large stretch behavior of rubber elastic materials. Journal of the Mechanics and Physics of Solids, 1993, 41 (2), pp.389-412. 10.1016/0022-5096(93)90013-6 . hal-01390807

\section{HAL Id: hal-01390807 https://hal.science/hal-01390807}

Submitted on 2 Nov 2016

HAL is a multi-disciplinary open access archive for the deposit and dissemination of scientific research documents, whether they are published or not. The documents may come from teaching and research institutions in France or abroad, or from public or private research centers.
L'archive ouverte pluridisciplinaire HAL, est destinée au dépôt et à la diffusion de documents scientifiques de niveau recherche, publiés ou non, émanant des établissements d'enseignement et de recherche français ou étrangers, des laboratoires publics ou privés. 


\title{
A THREE-DIMENSIONAL CONSTITUTIVE MODEL FOR THE LARGE STRETCH BEHAVIOR OF RUBBER ELASTIC MATERIALS
}

\author{
Elitin M. Arruda and Mary C. Boyct. \\ The Department of Mechanical Fngineering. The Massachusetts Institute of Technology. \\ Cambridge, $\mathrm{M} \Lambda$ (22139. U.S.A.
}

\begin{abstract}
ABS TRAC"T
A constirl "rive model is proposed for the deformation of rubber materials which is shown to represent successfully the response of these materials in uniaxial extension, biaxial extension, uniaxial compression. plane strain compression and pure shear. The developed constitutive relation is based on an eight chain representation of the underlying macromolecular network structure of the rubber and the non-Gaussian behavior of the individual chains in the proposed network. The eight chain model accurately captures the cooperative nature of network deformation while requiring only two material parameters. an initial modulus and a limiting chain extensibility. Since these two parameters are mechanistically linked to the physics of molecular chain orientation involved in the deformation of rubber, the proposed model represents a simple and accurate constitutive model of rubber detormation. The chain extension in this network model reduces to a function of the root-mean-square of the proncipal applied stretches as a result of effectively sampling eight orientations of principal stretch space. The results of the proposed eight chain model as well as those of several prominent models are compared with experimental data of TrFiofor (1944. Trums. Faraday. Soc, 40, 59) illustrating the superiority, simplicity and predictive ability of the proposed model. Additionally, a new set of experiments which captures the statc of deformation dependence of rubber is described and conducted on threc rubber materials. The eight chain model is found to model and predict accurately the behavior of the three tested materials further confirming its superiority and effectiveness over earlicr nrodels.
\end{abstract}

\section{IVTRODUCTION}

THE AIM OF THIS WORK has been to develop a fully three-dimensional. mechanistically motivated constitutive relationship for nonlinear elasticity which would successfully account for the state of deformation dependent response of rubber materials. The need for such a retationship is readily evident to any processor of rubber components or purveyor of rubber goods concerned with aspects of material behavior under large stretch deformation states which are not simple tension. A good constitutive model should represent the three-dimensional nature of the stress -stretch behavior using a minimal number of parameters to represent physically the deformation process. Ideany, the parameters should be obtainable from a small number, preferably onc, of experiments. Previous models of rubber elasticity such as WANi; and Guth (1952) and FLORY and RFHNFR (1943) have described or otherwise accounted for the characteristic ' $\mathrm{S}$ '-shaped load versus stretch curve exhibited by rubber materials in uniaxial 
tension. The load-stretch curve is highly dependent upon the state of imposed deformation, shown, for example, in the data of TrELOAR (1944) depicted in Fig. 1. The authors know of no existing model which accurately represents the behavior of such materials in various deformation states and satisfies the criterion of requiring only a small number of "physically based" parameters. Such material parameters or constants should be independent of deformation state in order to provide a predictive capability to the constitutive model.

The first statistical mechanics approach to describing the force on a deforming polymeric network assumed Gaussian statistics to apply, that is the chains never approached their fully extended length $r_{\mathrm{L}}=I N$ where $N$ is the number of statistical links of length $l$ in the chain between chemical crosslinks. [See TrELOAR (1975) for instance for a more detailed description of Gaussian statistics and the corresponding assumptions.] Gaussian statistics yields

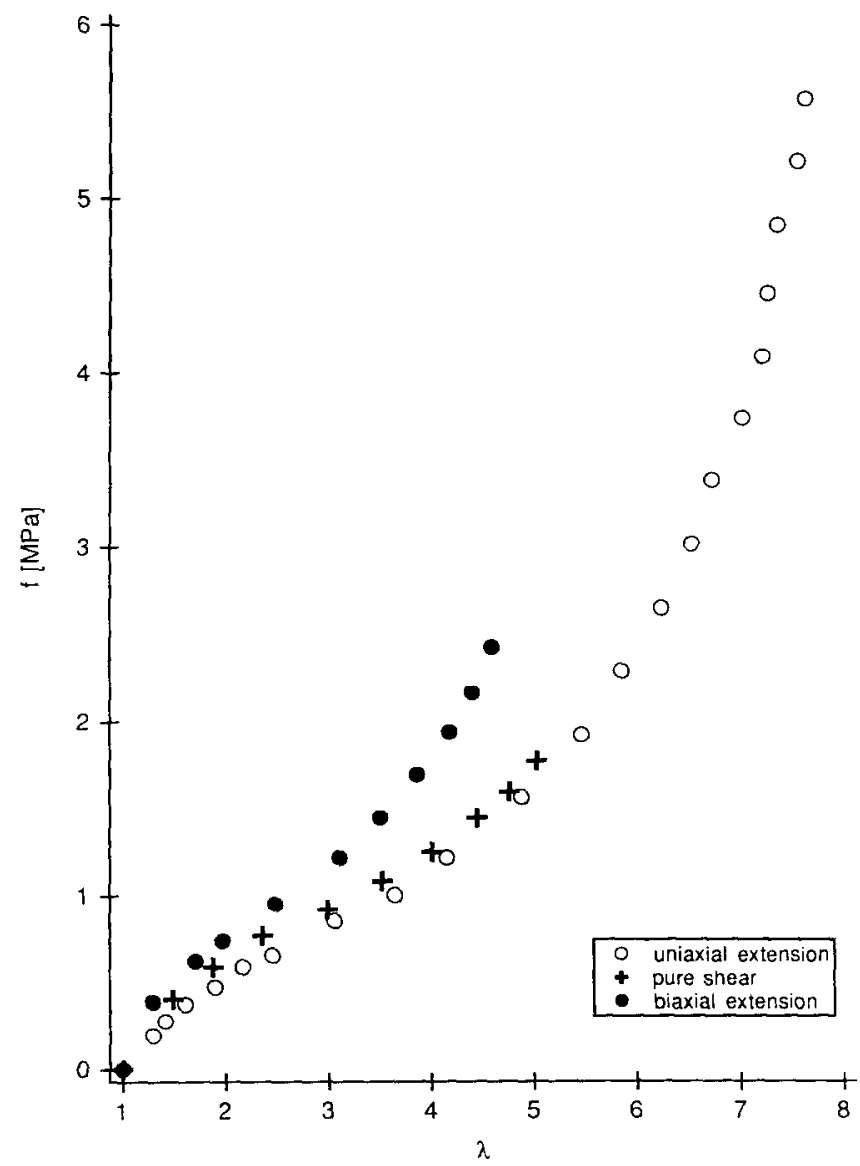

Fig. 1. Data from Treloar (1944), plotted as force per unit unstrained area versus stretch, showing the state of deformation dependence of a rubber material in uniaxial extension, biaxial extension and shear. 


$$
W=\frac{1}{2} G\left(\lambda_{1}^{2}+\lambda_{2}^{2}+\lambda_{3}^{2}-3\right), \quad G=n k \Theta
$$

for the strain energy where the $\lambda_{i}$ are the applied stretches and the rubbery modulus $G$ is a function of the chain density, $n$, Boltzmann's constant, $k$ and temperature $\Theta$.

Investigators since have built networks from chains described by Gaussian statistics or have modified the chain statistics to allow larger stretches than are afforded by the assumption of Gaussian statistics, then incorporated these non-Gaussian chains into networks of three, four or an infinite number of chains. See WANG and GUTH (1952), FLORY and REHNER (1943) and TRELOAR (1946) for the three chain non-Gaussian, four chain Gaussian and four chain non-Gaussian networks, respectively, or TRELOAR (1975) for an overview of network models including his own proposed method of averaging the contribution of a single chain over a large number of orientations. $\dagger$ These models have in common two physically based parameters, the rubbery modulus $G$ given in (1) and a chain locking stretch $\lambda_{L}$ defined as the value of the chain stretch when the chain length reaches its fully extended state. Chain stretch is always given by the current chain length divided by the initial chain length

$$
\lambda_{\text {chain }}=\frac{r_{\text {chain }}}{r_{0}^{-}} \text {. }
$$

The initial chain length is given from random walk statistics as $r_{0}=\sqrt{N} l$. Thus for the fully extended or locking chain length, $r_{L}=l N$, the locking stretch becomes

$$
\hat{\lambda}_{\mathrm{L}}=\frac{r_{\mathrm{L}}}{r_{0}}=\sqrt{N}
$$

Other statistical models have investigated affine versus phantom deformation of junctions in networks and these have been discussed by MARK and ERMAN (1988). In many cases models such as these require a third or fourth parameter to describe some measure of chain interaction in the network.

Excellent historical perspectives of the phenomenological invariant-based models of rubber deformation such as those of MoONEY (1940), RivLIN (1948), VALANIS and LANDEL (1967) and OGDEN (1972) have been given by Treloar $(1975,1976)$. The theories of this genre are aimed at obtaining an expression for the elastic strain energy which is of the form dictated by continuum mechanics as concerns an initially isotropic, incompressible, hyperelastic solid. The phenomenological theories lack a direct physical connection to the underlying mechanisms of deformation. The most sophisticated of these is that of OGDEN (1972) which proposes the following form for the strain energy function

$$
W=\sum_{n} \frac{\mu_{n}}{\alpha_{n}}\left(\lambda_{1}^{\alpha_{n}}+\lambda_{2^{1}}^{\alpha_{1}}+\lambda_{3^{\prime \prime}}^{\alpha_{1}}-3\right)
$$

with $\mu_{n}$ and the $\alpha_{n}$ as experimentally fitted constants. Ogden makes no attempt to link the adjustable parameters to any physical deformation mechanism. The Ogden model is essentially empirical and generally requires more than one experiment to obtain the number of constants required to capture state of deformation dependence.

† The averaging was later published in TRELOAR and RIDING (1979). 


\section{Proposed Model}

\subsection{Requirements of a model}

The existing models referenced above have merit and are frequently used in treating rubber deformation problems. Many of the early models capture the ' $S$ '-shaped load versus stretch curve exhibited by rubber materials in uniaxial tension. A relative dearth of experimental evidence exists in the literature for the response of rubbers in other deformation states up to very large stretches save for the work of TrEIoAR (1944), in uniaxial extension, biaxial extension and shear, considered by many to be the quintessential rubber data, $\uparrow$ and the extensive biaxial experiments of JoNEs and Treloar (1975). Consequently, many of the existing models fail in the task of describing the response of a rubber material under different states of deformation without changing the model parameters. In other cases the models are prohibitive in the number of parameters required to fit the data or the mathematical complexity they represent. The need for a constitutive relationship which possesses mathematical simplicity, requires one test to characterize the material and has a limited number of parameters, has prompted the authors' development of the following model.

\subsection{Langevin chain statistics}

The statistical mechanics approach to rubber elasticity models the rubber chain segment between chemical crosslinks as a number $N$ of rigid links of equal length $l$. The rigid link is that segment length of the actual chain which undergoes rigid body motion in response to an imposed strain, depending on the actual rubber material the statistical rigid link may span one or several repeat molecular units. The initial chain length is taken from a random walk consideration of $N$ steps of length $l$, and is denoted by $r_{0}$,

$$
r_{0}=\sqrt{N} l
$$

The fully extended chain has approximate length $I N$ so that the limiting extensibility (or chain locking stretch), defined as the final length divided by initial length, is given in terms of the statistical parameters as $\lambda_{\mathrm{L}}=\sqrt{N}$. At any value of chain length the most probable angular distribution of rigid links about the chain vector length may be found. Following the use of Langevin statistics by KUHN and GRÜN (1942), the probability of the most probable link angle distribution about a given vector length is taken to be equal to the probability of the vector length. The chain vector length is denoted by $r_{\text {chain }}$. In this way Kuhn and Grün obtain an expression for the probability density function for chain lengths and subsequently the configurational entropy of a stretched chain of current length $r_{\text {chan }}$

\footnotetext{
$\dagger$ Some debate has persisted over whether the characteristic upturn in the observed stress-stretch behavior is solely due to crystallization and therefore, not present in all rubbers. In the following section we offer additional experimental results on three rubber materials which exhibit the same basic, characteristic behavior as that of Treloar's data.
} 


$$
S_{\text {chain }}=k\left[c-N\left(\frac{r_{\text {chain }}}{N l} \beta+\ln \frac{\beta}{\sinh \beta}\right)\right],
$$

where $c$ is a constant, $k$ is Boltzmann's constant and $\beta$ is the inverse Langevin function, $\beta=\mathscr{L}^{-1}\left[r_{\text {chain }} / N l\right]$, for the Langevin function defined as $\mathscr{L}[\beta]=\operatorname{coth} \beta-(1 / \beta)$. The use of Langevin statistics properly accounts for the limiting chain extensibility. The work of deformation is proportional to the entropy change on stretching the chains from the unstretched state and may be written in terms of the chain length as

$$
W=n k \Theta N\left(\frac{r_{\text {chain }}}{N l} \beta+\ln \frac{\beta}{\sinh \beta}\right)-\Theta c^{\prime},
$$

where $n$ is the chain density, $c^{\prime}$ is a combination of constants and the remaining terms are as defined previously. The material is considered to be incompressible and the principal stresses can be determined from the work of deformation to within an arbitrary pressure, $p$,

$$
\sigma_{i}=\lambda_{i} \frac{\mathrm{d} W}{\mathrm{~d} \lambda_{i}}+p
$$

where the $\lambda_{i}$ are the principal stretches and the pressure may be determined from the boundary conditions. The stress-stretch relations are frequently written in terms of the difference in two principal stresses in order to eliminate the pressure term.

$$
\sigma_{1}-\sigma_{2}=\lambda_{1} \frac{\mathrm{d} W}{\mathrm{~d} \lambda_{1}}-\lambda_{2} \frac{\mathrm{d} W}{\mathrm{~d} \lambda_{2}} .
$$

\subsection{Use of Langevin chain networks}

The use of Langevin chain statistics in networks has been considered by WANG and GUTH (1952) in their three chain model by TRELOAR (1946) in his extension of the tetrahedron model of FLORY and REHNER (1943). TRELOAR (1975) and later TRELOAR and RIDING (1979) also considered a model of a large assembly of chains which were averaged by integrating over many spatial orientations. Analytical results of nonGaussian three chain, tetrahedron and total assembly of chains models are presented in Treloar (1975) for uniaxial tension. The three chain and tetrahedron models have been considered by the present authors for use as constitutive models of rubber deformation, results of simulations of different deformation states using both models will be presented later and compared to the proposed model. The three chain and tetrahedron model systems are sketched in Figs 2 and 3 respectively for undeformed, uniaxial extension and biaxial extension load geometries.

These models were also previously considered for their general predictive capabilities of the characteristic ' $S$ '-shaped uniaxial response by TRELOAR (1975). He observed that the response of the tetrahedron model was slightly dependent upon the orientation of the volume element with respect to the extension direction. In their 1952 paper, Wang and Guth stipulated that the faces of the three chain cube element be aligned with the principal stretch space during deformation. TRELOAR (1975) concluded that 
(a)

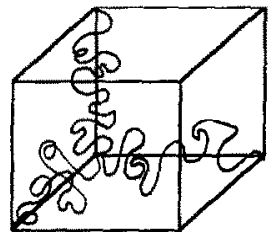

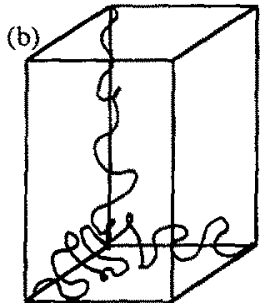

(c)

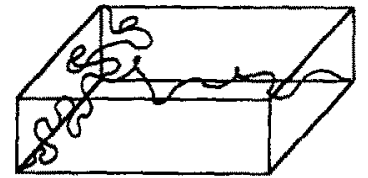

Fig. 2. Three chain rubber elasticity model for (a) undeformed, (b) uniaxial extension and (c) biaxial extension configurations.

these models possess an inherent anisotropy which could be eliminated by a system of a large assembly of chains, cach of length equal to the root-mean-square chain length, $r_{0}=\sqrt{N}$, which occupy random orientations in space. In this manner the contributions from chains in several orientations may be averaged to give the overall response. His proposed total assembly of chains model requires integration over the distribution of chains for each subjected stretch state, a procedure which was mathematically prohibitive 15 years ago and remains cumbersome today.

The authors agree with Treloar's assessments of the existing network models except for his reluctance to restrict the orientation of a model to some specific relationship with respect to principal stretch space as Wang and Guth had done. Motivated by Treloar's assertion to present a model which averages several spatial orientations, but recognizant of the need for a mathematically concise representation, we have proposed a model which possesses the cubic symmetry of principal stretch space as it averages eight orientations of that space in determining the network response. The clear advantage of this technique is its ability to simulate a true network response of cooperative chain stretching which presents a clear picture of the deformation process with mathematical ease.

\subsection{Proposed model geometry}

The proposed model considers eight orientations of chains in space which may be envisioned by the eight chain network system sketched in Fig. 4 for undeformed,

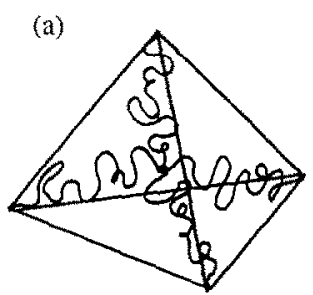

(b)

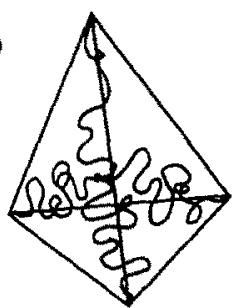

(c)

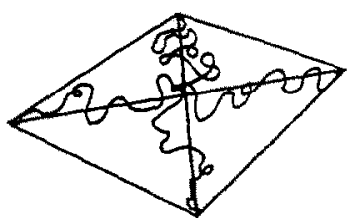

Fig. 3. Four chain rubber elasticity model for (a) undeformed, (b) uniaxial extension and (c) biaxial extension configurations. 
(a)

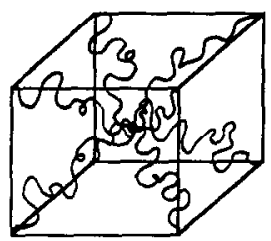

(b)

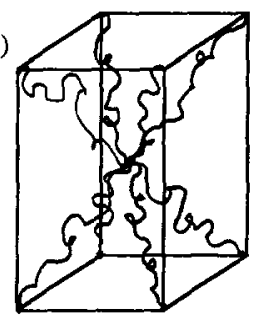

(c)

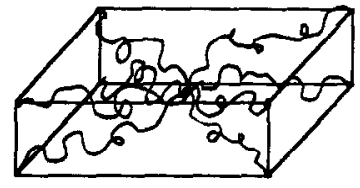

FiG. 4. Eight chain rubber elasticity model for (a) undeformed, (b) uniaxial extension and (c) biaxial extension configurations.

uniaxial extension and biaxial extension loaded configurations. The chain length, $r_{\text {chain }}$, is sought for each of the chains in the eight chain model as a function of the imposed principal global stretches $\lambda_{1}, \lambda_{2}, \lambda_{3}$ in order that (7) may be differentiated to obtain the stress-stretch relations (9). Consider the cube containing the eight chain configuration in Fig. 5. The cube edges are taken to remain aligned with principal stretch space during deformation, chains linked at the center of the cube extend to the eight corners. In principal space the cube is allowed expansion along each principal direction subject only to incompressibility which may be expressed as

$$
\lambda_{1} \hat{\lambda}_{2} \hat{\lambda}_{3}=1 \text {. }
$$

Note that the restriction to principal stretch space does not limit the usefulness of the model to axisymmetric deformations. For example, in pure shear the principal stretches are $\lambda_{1}=\lambda, \lambda_{2}=1$ and $\lambda_{3}=\lambda^{-1}$. The directions in which these stretches act rotate continuously with the deformation and this rotation is monitored by the standard kinematics of finite strain deformation making use of the polar decomposition and subsequent extraction of the stretch tensor eigenvalues from the general

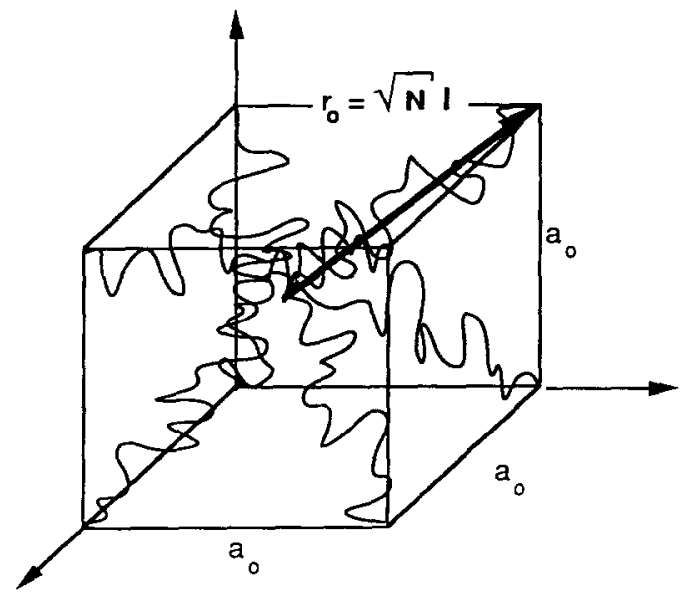

FIG. 5. The unstretched network for the proposed eight chain model. 
deformation gradient tensor. See for example the discussion by Hopkins in TreLOAR (1976) or FARDSHISHEH and ONAT (1972). The eight chain model is always stretched in the principal frame and is in general oriented differently from the laboratory frame. The rationale for use of the model in this manner follows from the consideration that in response to any deformation a principal stretch frame exists and the chains in that reference frame will undergo stretches describable by the principal values of stretch, $\lambda_{1}, \lambda_{2}$ and $\lambda_{3}$.

The unstretched network includes eight chains of length $r_{0}=\sqrt{N} l$ inside a cube of dimension $a_{0}$. From this geometry

$$
a_{0}=\frac{2}{\sqrt{3}} r_{0} .
$$

In Fig. 6 the cube is stretched by $\lambda_{1} \hat{i}, \lambda_{2} \hat{j}$ and $\lambda_{3} \hat{k}$ so that the cube edges measure $\lambda_{1} a_{0}$, $\lambda_{2} a_{0}$ and $\lambda_{3} a_{0}$ in the $\hat{i}, \hat{j}$ and $\hat{k}$ directions, respectively. A chain vector from the center of the cube to a corner may be written for one chain as

$$
\mathbf{c}_{1}=\frac{a_{0}}{2} \lambda_{1} \hat{i}+\frac{a_{0}}{2} \lambda_{2} \hat{j}+\frac{a_{0}}{2} \lambda_{3} \hat{k}
$$

This chain has vector length

$$
\left.r_{\text {shiuin }}=\frac{a_{0}}{2}\left(\lambda_{1}^{2}+\lambda_{2} \frac{2}{2}+\lambda_{3}^{2}\right)\right)^{1 / 2}
$$

as do all remaining chains in the given network geometry, regardless of deformation state. Substitution from (11) and (5) into (13) gives the chain length in terms of the statistical parameters and the principal stretches

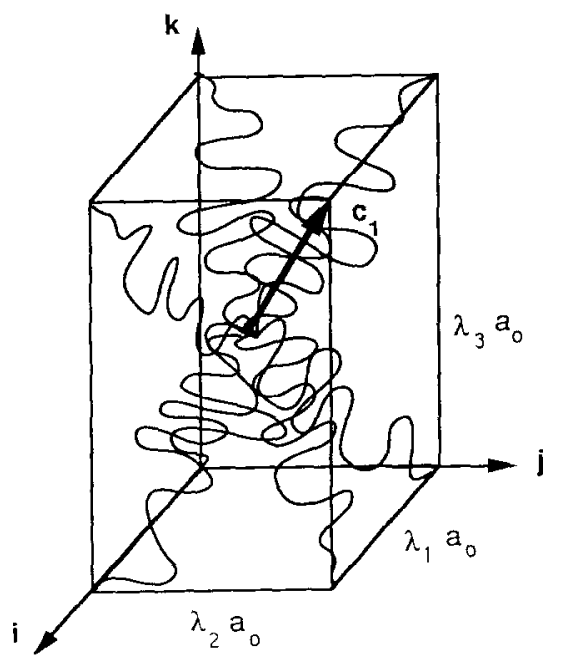

Fia. 6. The cight chain network in a stretched configuration. 


$$
r_{\text {chain }}=\frac{1}{\sqrt{3}} \sqrt{N} l\left(\lambda_{1}^{2}+\lambda_{2}^{2}+\lambda_{3}^{2}\right)^{1 / 2} .
$$

In this form the expression for the chain vector length is suitable for substitution into (7) which with (9) yields the following stress-stretch relation for the proposed model

$$
\sigma_{1}-\sigma_{2}=\frac{n k \Theta}{3} N \mathscr{L}^{-1}\left[\frac{\hat{\lambda}_{\text {chain }}}{\overline{\sqrt{N}}}\right] \frac{\left(\lambda_{1}^{2}-\lambda_{2}^{2}\right)}{\bar{\lambda}_{\text {chain }}},
$$

where the chain stretch, $\lambda_{\text {chain }}=r_{\text {chain }} / r_{0}$, is the same in each chain and is given by

$$
\lambda_{\text {chain }}=\frac{1}{\sqrt{3}}\left(\lambda_{1}^{2}+\lambda_{2}^{2}+\lambda_{3}^{2}\right)^{1 / 2} .
$$

Because each chain in the system undergoes a stretch equivalent to that in every other network chain, the model is likened to averaging the contributions of a single chain over eight spatial orientations.

The three stretch invariants are given by

$$
\begin{gathered}
I_{1}=\lambda_{1}^{2}+\lambda_{2}^{2}+\lambda_{3}^{2}, \\
I_{2}=\lambda_{1}^{2} \lambda_{2}^{2}+\lambda_{2}^{2} \lambda_{3}^{2}+\lambda_{1}^{2} \lambda_{3}^{2}, \\
I_{3}=\lambda_{1}^{2} \lambda_{2}^{2} \lambda_{3}^{2} .
\end{gathered}
$$

The expression for chain stretch is seen to reduce to a function of the first stretch invariant, $I_{1}$, and (16) may be rewritten as

$$
\lambda_{\text {chain }}=\frac{1}{\sqrt{3}} I_{1}^{1 / 2} .
$$

The strain energy of the proposed model may be found from integration of (15) using the series expansion form for the inverse Langevin function given for example in TRELOAR (1954). The first five terms for the strain energy of this model are

$$
\begin{aligned}
W=n k \Theta\left[\frac{1}{2}\left(I_{1}-3\right)+\right. & \left.\frac{1}{20 N}\left(I_{1}^{2}-9\right)+\frac{11}{1050 N^{2}}\left(I_{1}^{3}-27\right)\right] \\
& +n k \Theta\left[\frac{19}{7000 N^{3}}\left(I_{1}^{4}-81\right)+\frac{519}{673750 N^{4}}\left(I_{1}^{5}-243\right)\right]+\cdots .
\end{aligned}
$$

Note that the strain energy in (21) exhibits a nonlinear $I_{1}$ dependence as a result of the chain stretch being defined in terms of $I_{1}$ only.

\section{Experimental Procedure}

The uniaxial compression test and plane strain compression test represent near extremes in the behavior of polymeric networks under biaxial deformation states. 


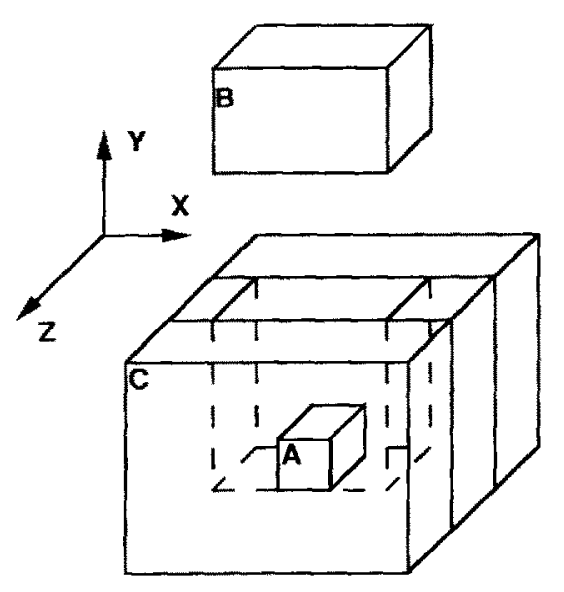

Fig. 7. Sketch of plane strain compression testing apparatus. Specimen (A) is compressed by punch (B) in the $Y$-direction and expands in the $X$-direction. The die (C) constrains the specimen along the $Z$-axis.

During uniaxial compression the polymer chains in the material stretch freely in all directions within a plane perpendicular to the load axis. Under plane strain compression the specimen is held within a channel die which completely constrains the material in one direction while allowing expansion in a second direction perpendicular to the first. The material flows along the expansion direction as a result of the compressive load applied perpendicular to the plane containing the constrained and expansion directions. In the plane strain compression die apparatus sketched in Fig. 7 , expansion occurs along the $X$-axis, the material is constrained along the $Z$-axis and $Y$ is the load direction. Polymer network stretching occurs only along the axis of free expansion in plane strain. The resulting stress versus stretch curve in plane strain compression differs from that in uniaxial compression because of the vastly different chain stretch behaviors in these two compression geometries.

Three commonly available rubber materials were chosen for this study: silicone rubber ( 40 durometer), neoprene nubber (60 durometer) and gum rubber (hardness unknown) + Uniaxial cubes of length $12 \mathrm{~mm}$ and plane strain specimens measuring $12 \mathrm{~mm} \times 9 \mathrm{~mm} \times 12 \mathrm{~mm}$ were cut from these materials. In the plane strain compression tests the $9 \mathrm{~mm}$ dimension was constrained. Uniform deformations of the order of $-200 \%$ strain were achieved in uniaxial compression and $-150 \%$ strain in plane strain compression. The specimen (and die, in the case of plane strain) was placed between flat plates on the ends of the load strain on an Instron model 1350 servohydraulic testing machine. Liberal lubrication was applied on all specimen surfaces in contact with plates or die walls to insure uniform deformations; a $\mathrm{MoS}_{2}$ based paste made by Dow Corning was deemed sufficient for lubricating these materials. Linear ramp tests were performed on all three materials for both deformation states at displacement rates of approximately $1 \mathrm{~mm} \mathrm{~s}^{-1}$. The load and displacement output responses of the testing machine were converted to digital signals by a Kiethly $\mathrm{A} / \mathrm{D}$

t These three materials were obtained at the Greene Rubber Company of Cambridge, MA. 


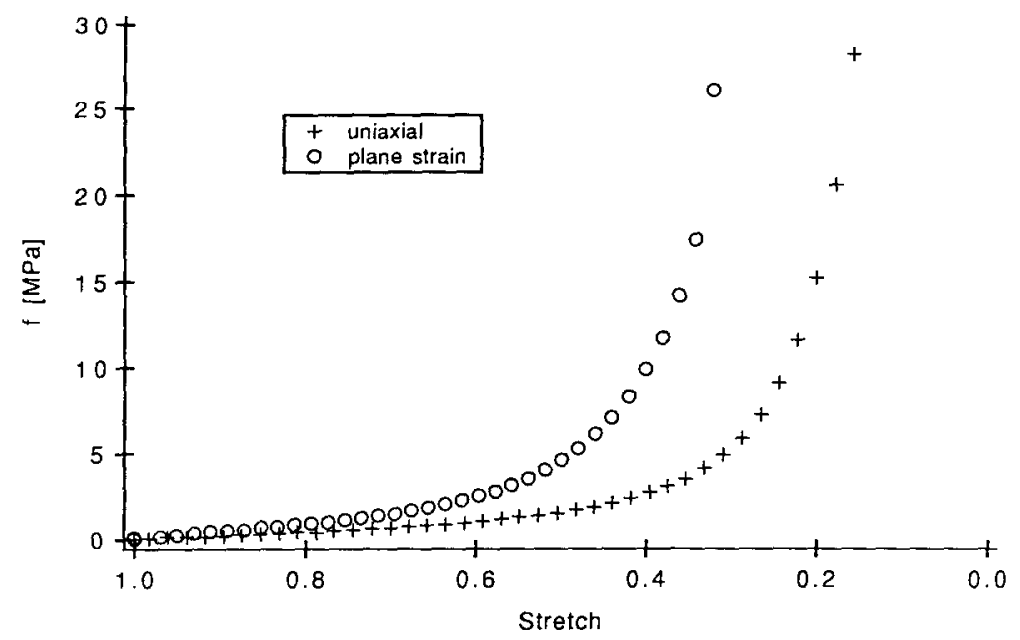

FlG. 8. Ramp tests in uniaxial compression and plane strain compression for silicone rubber.

board then transferred to a Macintosh personal computer for storage. The raw load versus displacement data were corrected for the testing machine compliance before being converted to nominal load (that is, load divided by initial area) versus stretch information. The results of ramp tests in uniaxial compression and plane strain compression are presented in Figs 8,9 and 10 for silicone, gum and neoprene rubber, respectively. Each of the three materials shows the basic characteristic response of a limiting stretch which is dependent upon the state of deformation. The three materials differ significantly in their initial moduli and/or locking stretch values.

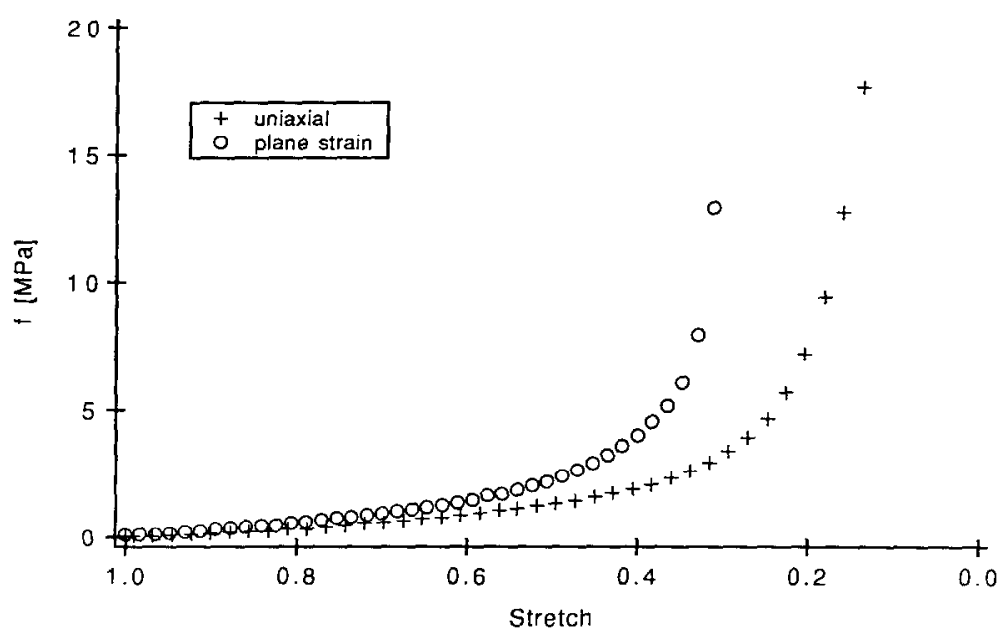

FiG. 9. Ramp tests in uniaxial compression and plane strain compression for gum rubber. 


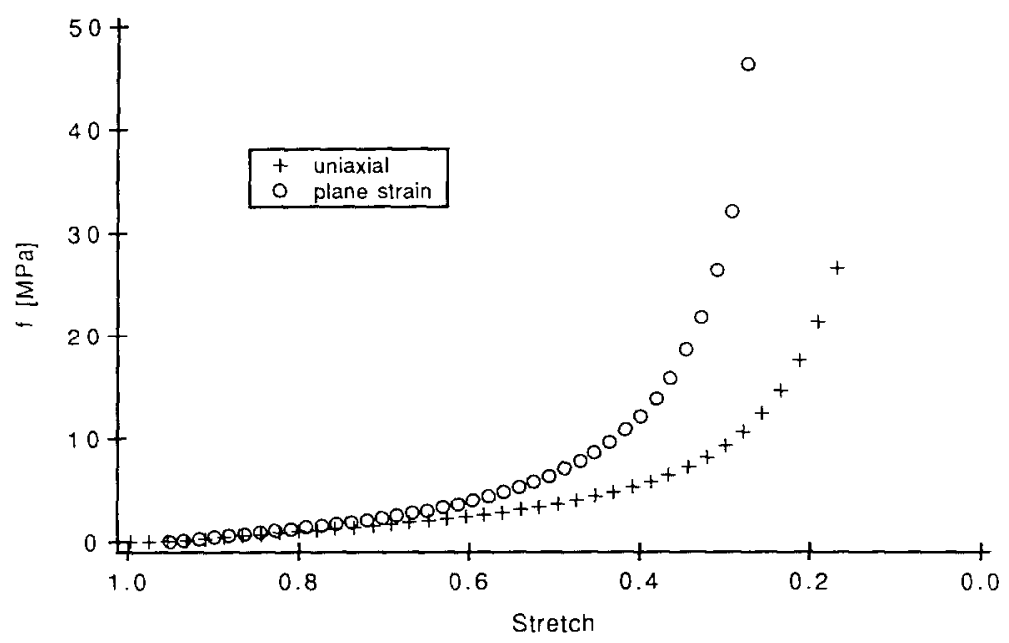

FIG, 10. Ramp lests in uniaxial compression and plane strain compression for neoprene rubber.

\section{RESUlTS}

\subsection{Explanation of the experimental data}

As shown earlier in Fig. 1, the data on Treloar's vulcanized rubber include tests in uniaxial extension, biaxial extension and pure shear to very large extension ratios and are plotted as load normalized by initial area versus stretch. The strong state of deformation dependence of the large and limiting stretch behavior of the polymeric network materials is shown in the data of Figs $1,8,9$ and 10 and remains a primary obstacle to true characterization of rubber elasticity. Additionally, ARRUDA and BOYCE (1991) have also reported a similar state of deformation dependent strain hardening response in glassy polymeric materials. These data on several different polymeric materials, both above and below the glass transition temperature, clearly demonstrate a certain level of generality to the presence of limiting stretch states in polymeric materials whether rubbery or glassy.

The equi-biaxial extension data in Fig. 1 varies significantly from either the uniaxial extension or pure shear data. The divergence of the biaxial data is due to the nature of the molecular chain stretching in equi-biaxial extension versus uniaxial extension. Biaxial extension offers two venues of principal tensile stretch $\left(\lambda_{1}=\lambda, \lambda_{2}=\lambda_{\text {. }}\right.$ $\lambda_{3}=\lambda^{-2}$ ); an initially isotropic network of chains will reach limiting chain extension due to stretching in both directions providing a planar state of orientation. In uniaxial tensile deformation the chains extend along one direction only $\left(\lambda_{1}=\lambda_{1} \lambda_{2}=\lambda^{1} \cdot 2\right.$, $\hat{\lambda}_{3}-\lambda^{-1 / 2}$ ), additional stretch is thus allotted through the drawing of material from the transverse directions, and the onset of limiting chain extension is delayed with respect to the biaxial deformation locking. The pure shear data are plotted in terms of the maximum principal stretch, $\lambda_{1}$, vs the corresponding normal force (i.e. not the shear force) which acts in the direction of $\lambda_{1}$. Pure shear deformation is more closely related to uniaxial extension than to equi-biaxial extension because chain stretch 
occurs due to stretching along one principal direction $\left(\lambda_{1}=\lambda_{2}, \lambda_{2}=1, \lambda_{3}=\lambda^{-1}\right)$ with chains being drawn from one direction transverse to the extension direction in pure shear. Thus a pure shear experiment yields a limiting stretch value which is similar to that obtained in uniaxial extension. A good model should be able to capture the observed differences between biaxial and uniaxial tensile behavior as well as the similarities in behavior between uniaxial and shear tests.

The plane strain compression and uniaxial compression data in Figs 8, 9 and 10 diverge for reasons similar to those used to explain the biaxial extension divergence from the uniaxial extension and pure shear data above. Uniaxial compression offers two directions of equal principal stretch $\left(\lambda_{1}=\lambda, \lambda_{2}=\lambda^{-1 / 2}, \lambda_{3}=\lambda^{-1 / 2}\right)$, whereas in plane strain compression chains extend along one direction only. The plane strain compression stretch state is equivalent to that of pure shear $\left(\lambda_{1}=\lambda, \lambda_{2}=1, \lambda_{3}=\lambda^{-1}\right)$.

\subsection{Explanation of simulations}

Equation (15) has been used together with the appropriate boundary conditions to simulate the uniaxial extension, biaxial extension and shear reponses of the proposed model. Characterization of a particular material requires determination of two parameters, the initial modulus and limiting extensibility, which is accomplished by fitting the model to the data for any one deformation state. The authors chose to fit Treloar's uniaxial extension data which yiclded the following parameters for this material: $C R=0.09$ and $N=26.5$. These parameters were used in the simulations of biaxial extension and pure shear tests. The results appear in Fig. 11. Using constants determined from this single set of uniaxial extension data the model quite accurately predicts the biaxial extension and pure shear data. These results demonstrate the predictive and fully three-dimensional aspects of our proposed eight chain model.

This exercise was repeated for both the three chain model of WANG and GUTH (1952) and the non-Gaussian tetrahedron model as modified by Treloar (1946, 1954). The stress-stretch relations for the Wang and Guth model are of the type

$$
\sigma_{1}-\sigma_{2}=\frac{n k \Theta}{3}-\sqrt{N}\left(\hat{\lambda}_{1} \mathscr{L}^{-1}\left\{\begin{array}{c}
\lambda_{1} \\
-\sqrt{N}
\end{array}\right\}-\lambda_{2} \mathscr{L}^{-1}\left\{\begin{array}{c}
\lambda_{2} \\
\sqrt{N}
\end{array}\right\}\right)
$$

where the parameters have the same meaning as in (15). The numerical treatment used for the tetrahedron model followed the early method of TRELOAR (1954) which allowed the central junction point to seek an equilibrium position for affine displacements of the tetrahedron corners. Equilibrium was found using an iterative Newton scheme after each stretch increment for the condition of no net force on the junction point. Stretch-stress relations are a function of the junction point equilibrium position and must be determined numerically. Each of these models requires one set of data to determine an initial modulus and locking stretch. The constants were found by fitting the models to the uniaxial extension data then used in predicting the biaxial extension and pure shear responses. The results for the three chain model are given in Fig. 12 and for the tetrahedron model in Fig. 13; each figure includes the values used for the model parameters. As these figures clearly show, the early models do not 


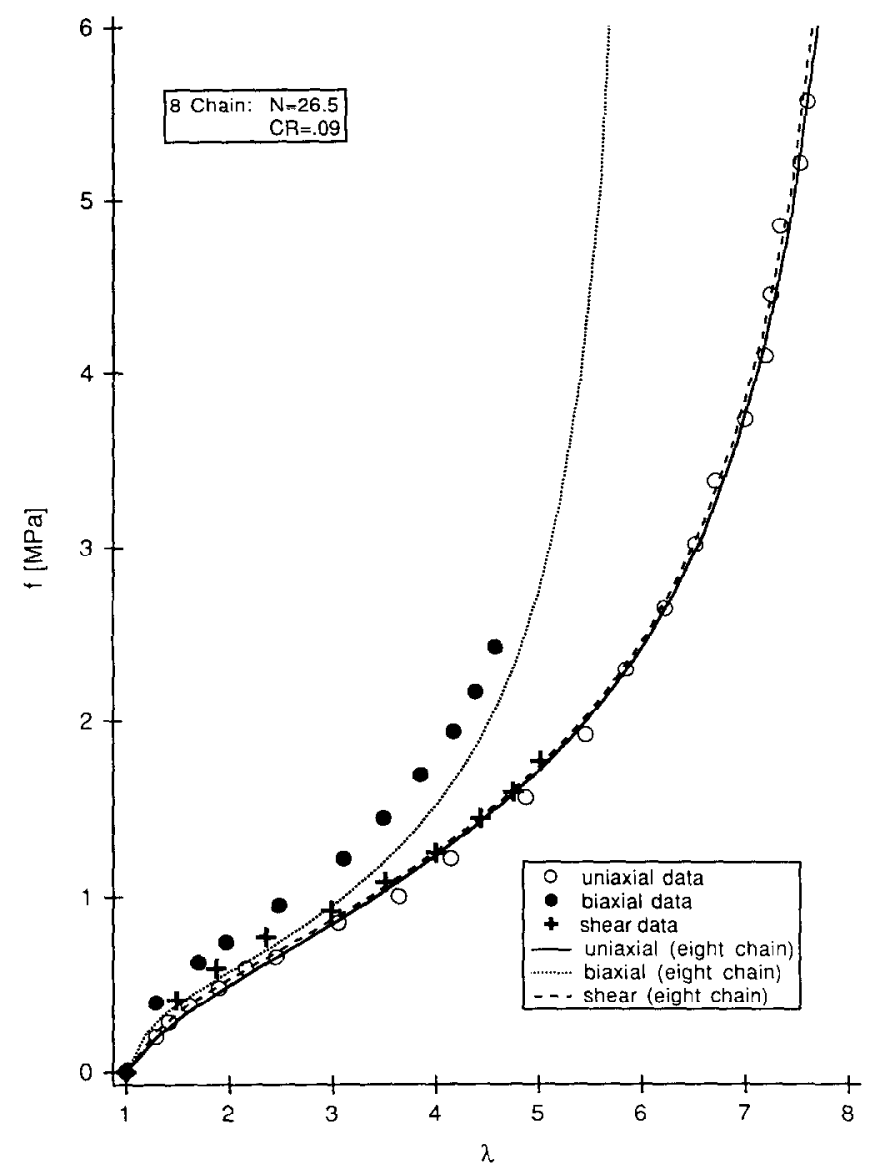

FiG. 11. Results of simulations using the eight chain model versus data from TreloAR (1944) in uniaxial extension, biaxial extension and shear.

predict much of a state of deformation dependence beyond the initial modulus and cannot be used as constitutive indicators of rubber elastic deformation.

The three chain model shows no state of deformation dependence beyond a stretch of 4.0 for the three stretch states examined; the results of the simulations for three states of deformation converge at large stretches whereas the data diverge. The failure of the three chain model to capture the state of deformation dependence lies in the absence of a cooperative nature of network chain deformation in this model except through the imposition of incompressibility. In uniaxial extension, depicted in Fig. 2, the chain parallel to the extension direction stretches until it reaches the locking stretch. The remaining two chain stretches are determined by incompressibility and do not contribute significantly to the force. Under equi-biaxial extension, two coplanar chains extend independently until simultaneously reaching the locking stretch and the third chain stretch is determined by consideration of incompressibility. Therefore the 


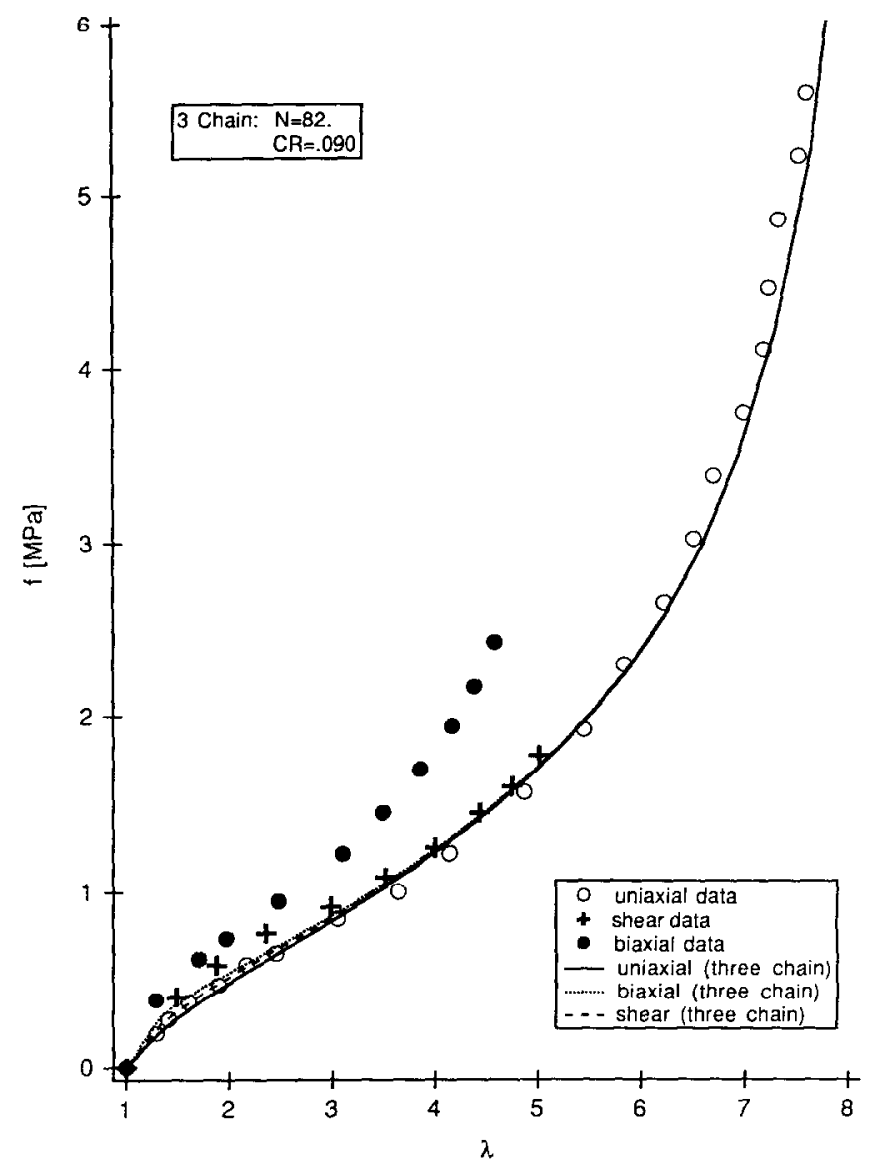

FIG. 12. Results of simulations using the Wang and Guth model versus data from TreloAR (I944) in uniaxial extension, biaxial extension and shear.

limiting stretch in equi-biaxial extension as predicted by the three chain model has the same value as that obtained in uniaxial extension and in pure shear, the result is convergence of the load vs stretch predictions for the three different stretch states rather than the actual divergence of these curves which the data show.

The tetrahedron model shows a slight state of deformation dependence at small stretches which is retained at large stretches. This model has some success in predicting a state of deformation dependence because the chains in this network do respond cooperatively to the deformation, see Fig. 3. Four chains stretch along the extension direction in uniaxial extension resulting in an overall network limiting tensile stretch which exceeds the locking stretch of a single chain $\left(\lambda_{\mathrm{L}}=\sqrt{N}\right)$. In biaxial extension perpendicular to one chain in the tetrahedron the remaining three chains undergo stretching. The result is again increased network extensibility over the single chain limiting stretch to a value which differs from the tensile locking stretch. The different values for locking in uniaxial and biaxial extension are manifested in the load-stretch 


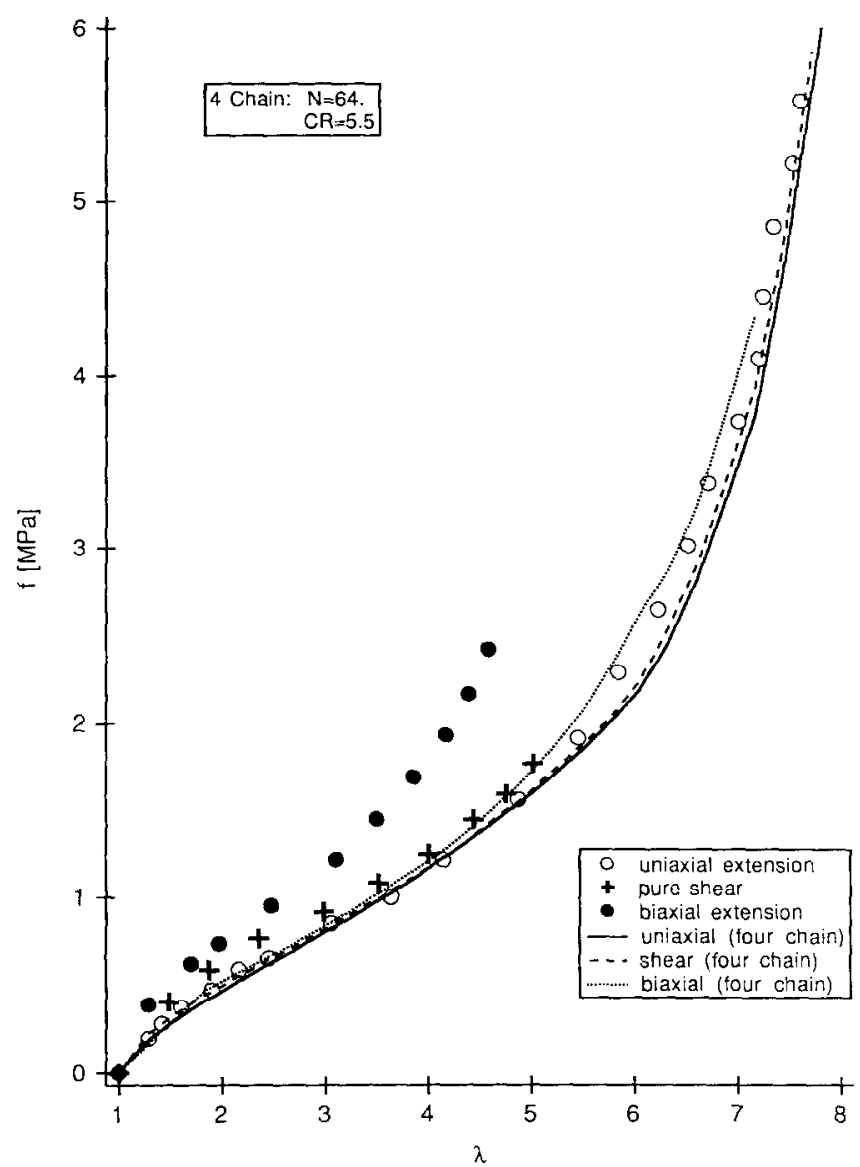

FIG. 13. Results of simulations using the four chain model versus data from TreloAR (1944) in uniaxial extension, biaxial extension and shear.

predictions of the four chain model which reach asymptotes at different values of stretch for different states of deformation.

The tetrahedron model does not possess symmetry with respect to the principal stretch space. The load versus stretch predictions depend upon the orientation of the four chain tetrahedron with respect to $\lambda_{1}, \lambda_{2}$ and $\lambda_{3}$ in addition to the magnitudes of the $\lambda_{i}$.

The eight chain model is clearly superior to the other statistical models in the state of deformation dependence predicted. The advantages of the eight chain model are its proximity in kind to a system which is initially isotropic, the use of Langevin chains which capture the effects of limiting chain extensibility and a network configuration which responds cooperatively to an imposed deformation. These features allow for mechanically simulating the state of deformation dependence of the behavior during large deformation while retaining mathematical simplicity. The features of the eight chain model as seen in Fig. 4 combine the favorable aspects of the previous three 
chain and four chain network models. The model possesses symmetry with respect to the principal stretch space which was required by WANG and GUTH (1952) in their discussion. All eight chains stretch uniaxially in response to an imposed uniaxial extension deformation as is true for the four chains in the FLORY-REHNER (1943) model. Again the limiting network stretch of the eight chain network exceeds the extensibility of a single chain in extension. In biaxial extension all eight chains extend identically due to stretching in two principal directions. The resulting limiting stretch is something other than either the individual chain locking stretch or the limiting stretch in uniaxial extension. A significant feature of the eight chain model is that all chains stretch equally under biaxial extension as well as uniaxial extension. The chain stretch is always the root-mean-square of the global principal stretch state. This unique feature of the eight chain model is true for all other deformation states; all chains stretch equally in response to any given deformation state.

Additional existing models were similarly tested against Treloar's data. These results are discussed in the Appendix.

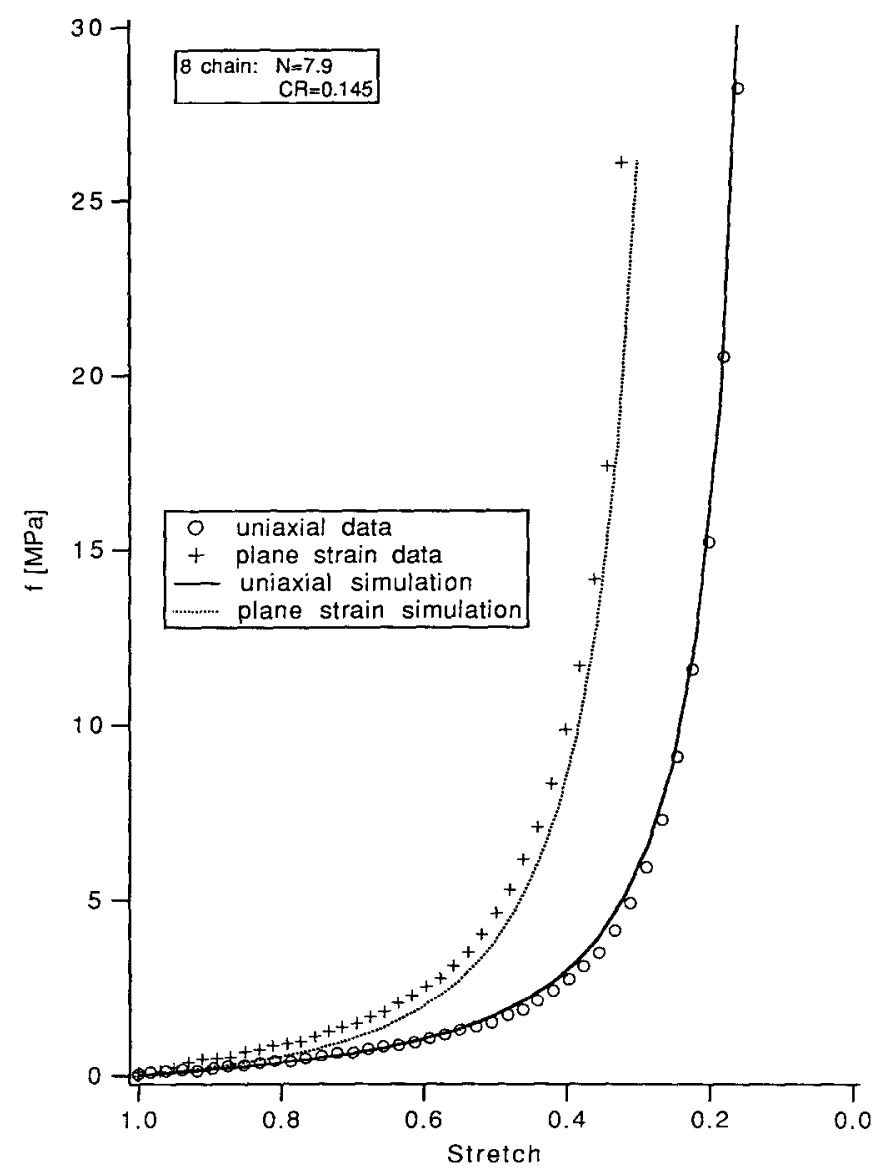

FIG. 14. Results of simulations using the eight chain model and data on silicone rubber. 


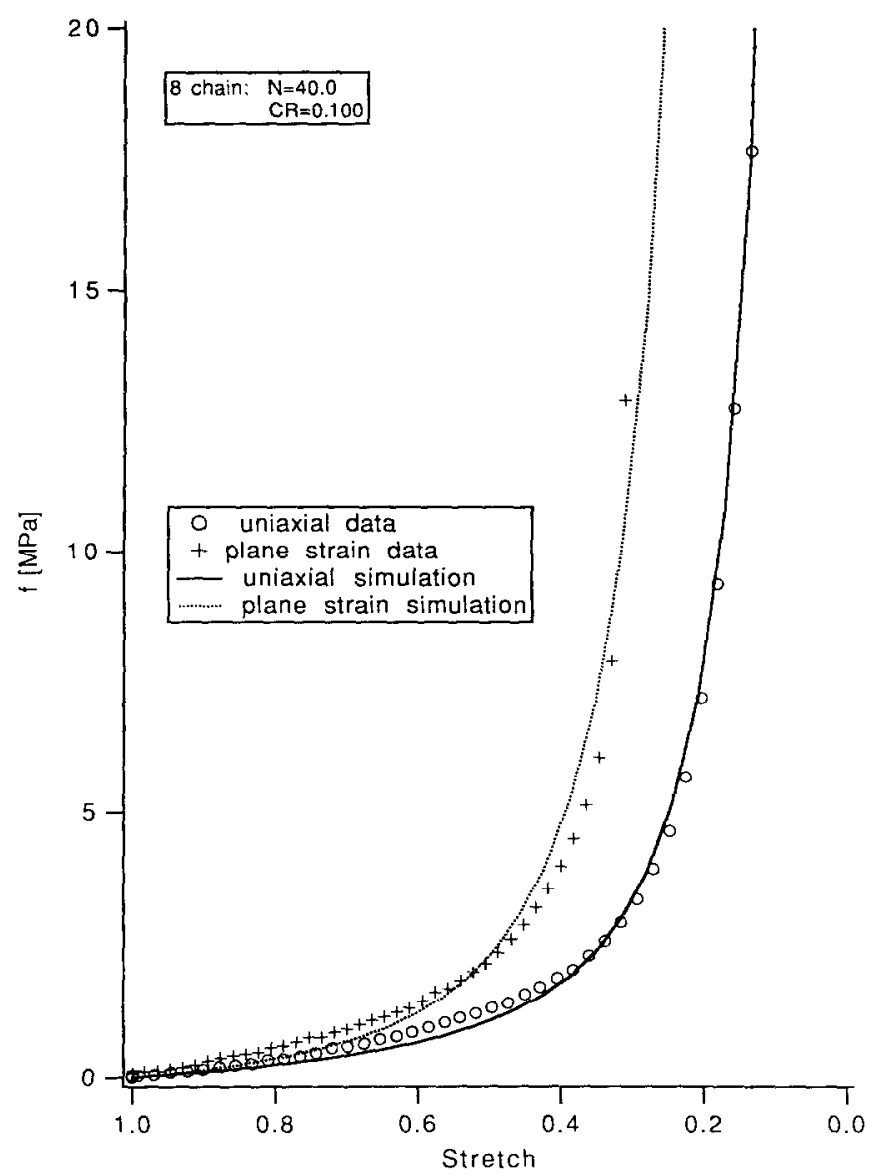

Fig. 15. Results of simulations using the eight chain model and data on gum rubber.

The proposed model was used to predict the plane strain compression responses of the three materials examined in this study based on the constants derived from their corresponding uniaxial compression responses. Results of these simulations appear in Figs 14, 15 and 16 for the silicone, gum and neoprene rubber materials previously described. The model parameters used to fit the uniaxial compression data are listed for each material on the corresponding figure. The model accurately captures the state of deformation dependence of all three materials. Each of these materials differs in the modulus and/or locking stretch value needed by the model to fit the uniaxial compression response. A direct comparison of the uniaxial compression responses of the three materials has been made in Fig. 17, here the differences in actual material moduli and limiting extensibilities are easily seen. Including Treloar's data the model has been shown to characterize four materials representing a range of material properties. Previous network models considered contained the same basic parameters of an initial modulus and a measure of finite extensibility, but were unable to predict a 


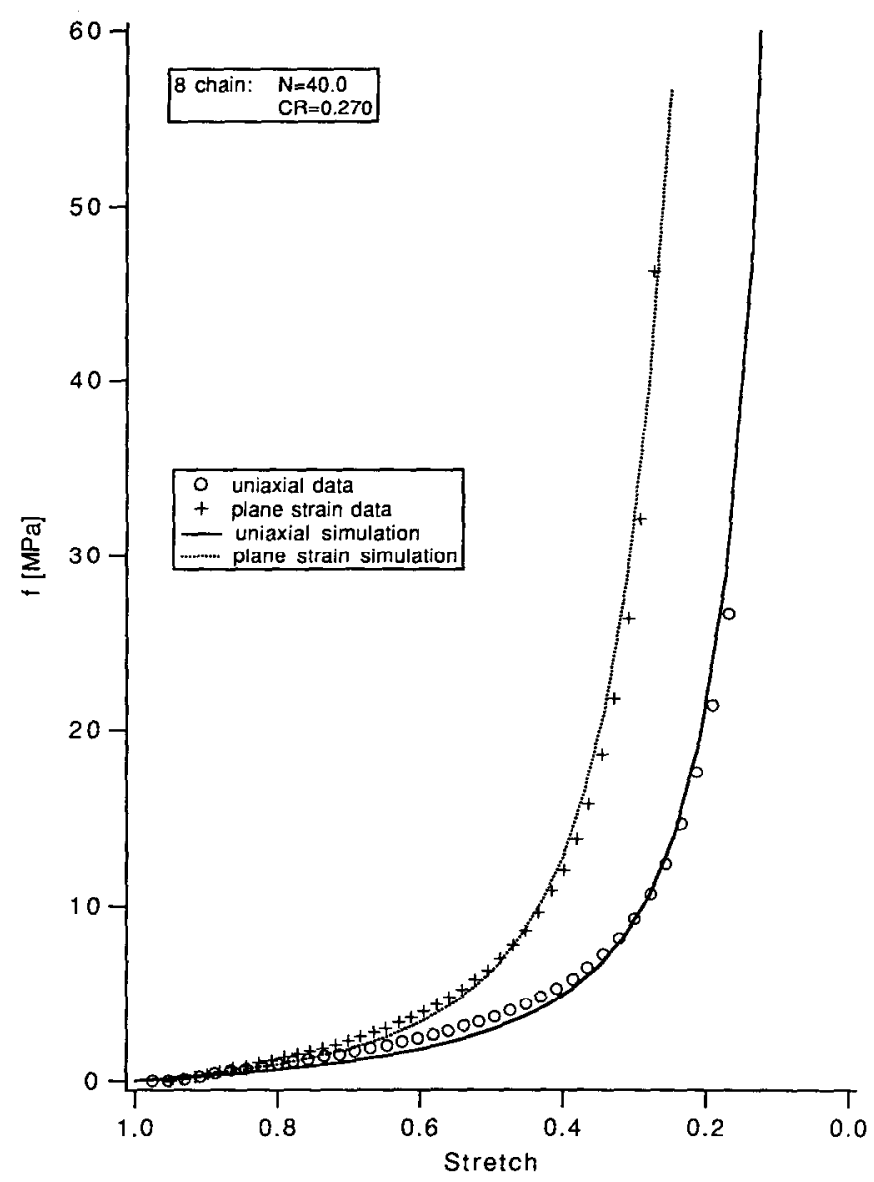

Fig. 16. Results of simulations using the eight chain model and data on neoprene rubber.

state of deformation dependence because of inabilities of the models to effectively represent a network response. In the case of the eight chain model the successful prediction is a result of correct usage of the finite extensibility parameter as that of an averaged measure of applied stretches over eight spatial orientations. The mathematically tractable eight chain model of (15) represents a true constitutive relationship for rubber deformation which is able to correctly account for the large stretch deformation response in one state of deformation, then with that characterization, predict other states of deformation.

A polymer below its glass transition temperature exhibits the same network response in strain hardening as rubber materials do during large stretch deformations. ARRUDA and BOYCE (1991) have shown the eight chain model to be successful in predicting the state of deformation dependence of the strain hardening response of glassy polymers whereas the three chain and tetrahedron models again fail. 


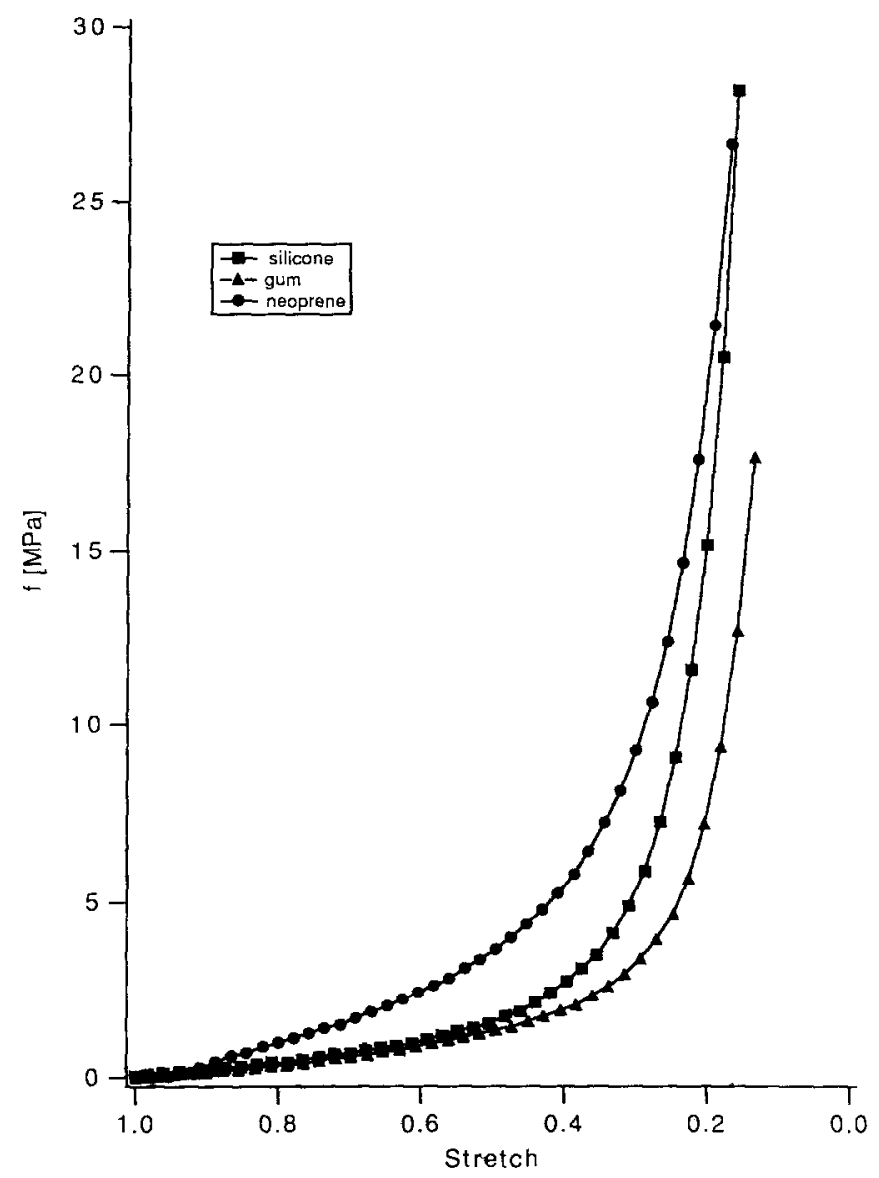

Fig. 17. Direct comparison of the uniaxial compression responses of silicone, gum and neoprene rubbers.

\section{Summary}

A physically based constitutive model for large stretch rubber deformation has been proposed which has been specifically designed to account for the three-dimensional state of deformation dependence in networked solids. The eight chain model presented here is formulated such that the nature of the state of deformation dependence is clearly seen to be the result of a network of chains reaching the individual chain extensibility limit at different imposed global stretch levels for different stretch states. The eight chain model successfully accounts for the state of deformation dependence using a rubbery modulus and a locking stretch as its only two parameters, both of which can be determined from a single experiment. Present in all statistical models, these parameters are physically linked to the polymeric network and therefore provide a basis for including other aspects of rubber elastic behavior such as temperature dependence, swelling and Mullin's effect. Indeed, the rubbery modulus term explicitly includes temperature. 
Experiments were conducted on three rubber materials to illustrate the state of deformation dependence of these materials and to determine the predictive capability and effectiveness of the proposed model. In addition the model was compared to existing rubber elasticity models in the ability to capture the response of data in the literature (TREIOAR, 1944) in three states of deformation. The eight chain model was shown to be superior in its overall ability to successfully account for the threedimensional nature to the underlying mechanics of network solid deformation for a total of four materials representing a range in material hardness and extensibilities.

The eight chain model contains many attractive features of concern in modelling the complicated deformation procedures involved in the finite straining of amorphous polymeric solids. It retains mathematical feasibility as a two parameter model and also contains the ability to respond to an imposed deformation state in a manner which simulates the actual mechanism governing the state of deformation response of rubber materials.

\section{ACK.NOWLFIDGFMENTS}

This work is sponsored by The National Science Foundation (MSM-8818233) and the MIT Bradley Foundation.

\section{REFERENCLS}

Arruda, F. M. and Boyce, M. C. 1991

FARDSHISHH, F. and ONAT, E. T. 1972

FLORY, P. J. and ERMAN, B.

FLORY, P. J. and REHNi:R, J., JR, 1943

JONis, D. F. and TRLIOAR, L. R. G. 1975

KuHN, W. and GrüN, F.

Mark. J. E. and Erman, B.

MOONEY, M.

OGDEN, R. W

RIVI.IN. R. S.

TrFloar, L. R. G.

TRFLOAR, L. R. G.

TRFLOAR, L. R. G.

TRei.oAk, L. R. G.

TrtLonR, L. R. G.

Trei.oAR, L. R. G. and RIDING, G. 1979

VALANIS, K. C. and Landei, R. F. 1967

WANG, M. C. and GUTH, E.
Anisotropy and localization of plastic deformation, Proc. Plasticity '91, p. 483.

In Problems in Plasticity (edited by A. SAW(ZUK), p. 89. Noordhoff, Leyden.

Macromol. 15, 800.

J. Chem. Phys. 11, 512 .

J. Phys. D : Appl. Phys. 8, 1285.

Kolloid Z. 101, 248.

Rubberlike Elasticily A Molecular Primer. John Wiley, New York.

1940 J. appl. Phys. 11, 582.

1972 Proc. R. Soc. Lond. A 326, 565.

1948 Phil. Trans. R. Soc. Lond. A. 241, 479.

1944 Trans. Faraday Soc. 40, 59.

1946 Trans. Faraday Soc. 42, 83.

1954 Trans. Faraday Soc. 50, 881.

1975 The Physics of Rubber Elasticity. Oxford University Press, Oxford.

Proc. R. Soc. Lond. A. 351, 301.

Proc. R. Sec. L.ond. A. 369, 261.

J. appl. Phys. 38, 2997.

J. Chem. Phys. 20, 1144.

\section{APPINIDIX}

Additional models were considered for comparison with the eight chain model in their ability to reproduce the state of deformation dependence of TrELOAR's (1944) data. Two phenom- 
enological models were considered, the well known Mooney-Rivlin (1948) relation and the Ogden (1972) model. The strain energy in the Mooney-Rivlin relation is given as

$$
W=C_{1}\left(I_{1}-3\right)+C_{2}\left(I_{2}-3\right) \text {, }
$$

where $C_{3}$ and $C_{2}$ are constants. The strain energy expression for the Ogden model was given in the text of this manuscript. The results of simulations with both of these models appear in Fig. Al along with the constants needed for each model. The simulations with the Ogden model were taken directly from OGDEN (1972). A minimum of six independently adjustable parameters are required by the Ogden model to fit these three deformation states shown in Fig. Al; more than one deformation state may have been considered in determining the six constants as only the first four constants are necessary to produce the uniaxial extension result.

An additional statistical mechanics model of FLORY and ERMAN (1982) which accounts for chain interactions has been considered. The elastic strain energy of the network is found from the sum of phantom and constraint contributions

$$
W=W_{\mathrm{ph}}+W_{0,},
$$

where $W_{\mathrm{ph}}$ of phantom Gaussian chains is

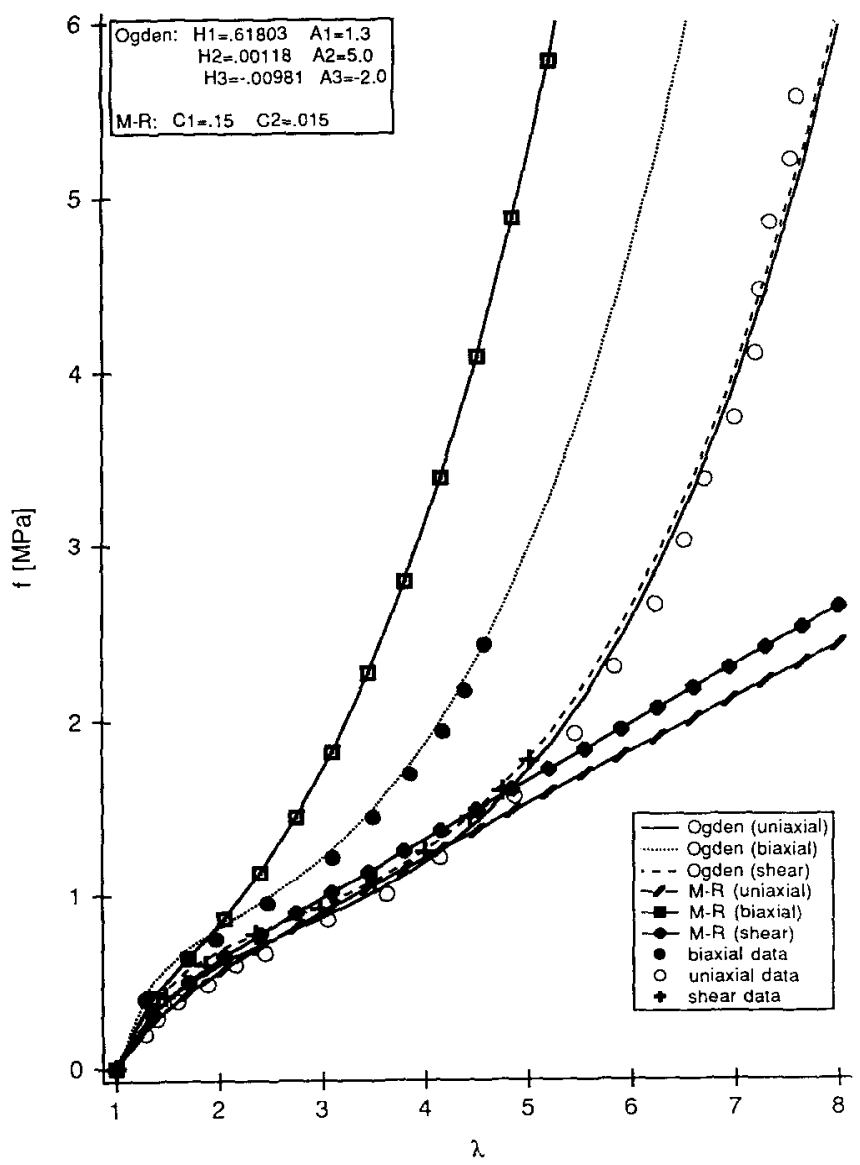

F1G. Al. Results of simulations using the Mooney-Rivlin and Ogden models versus data from TrELOAR (1944) in uniaxial extension, biaxial extension and shear. 


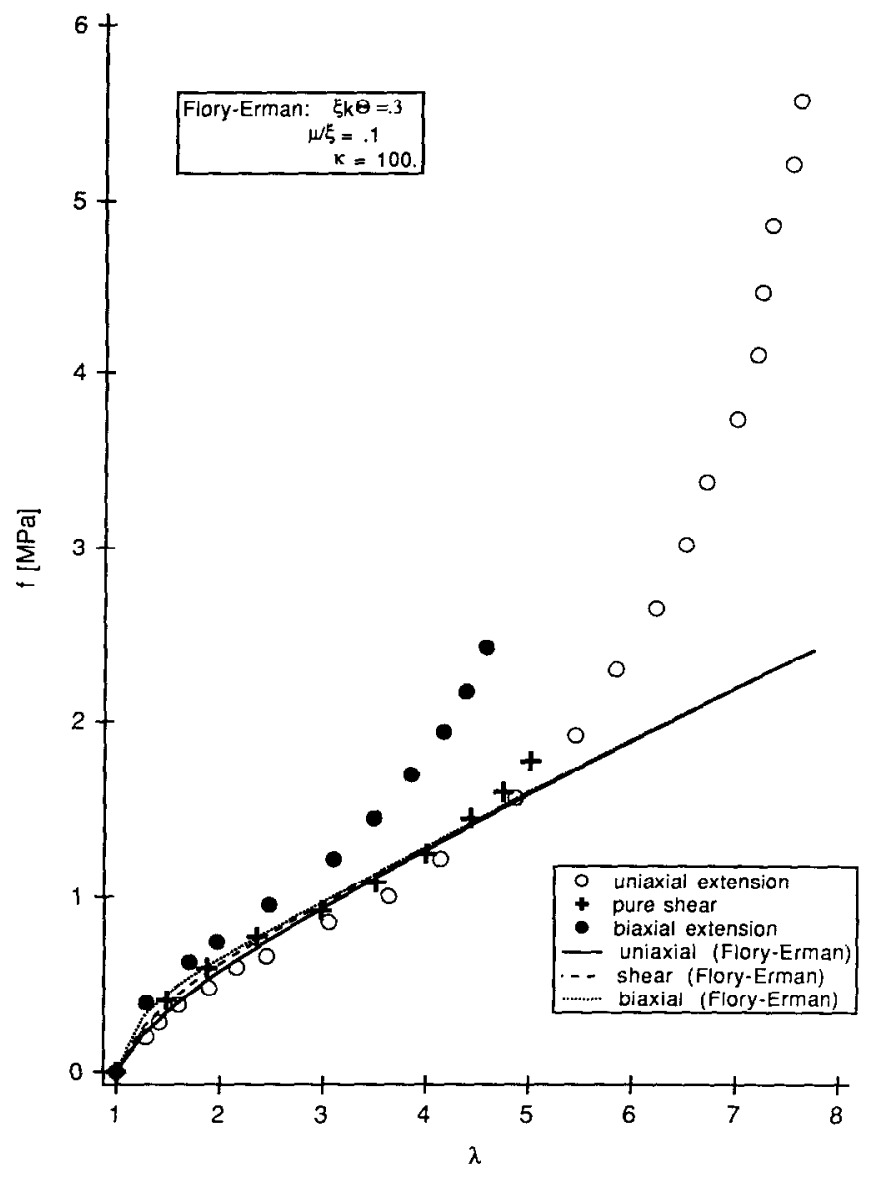

FIG. A2. Results of simulations using the Flory and Erman model versus data from TrELOAR (1944) in uniaxial extension, biaxial extension and shear.

$$
W_{\mathrm{ph}}=\frac{1}{2} \xi k \Theta\left(I_{1}-3\right)
$$

Note that (A3) differs from (1) in the text by the parameter $\xi$ where

$$
\xi=\left(1-\frac{2}{\phi}\right) n
$$

The parameter $n$ is the number of network chains and $\phi$ is the number of chains meeting at a junction. When $\phi=4$ the junction is tetrafunctional and the strain energy of the phantom network in (A3) is one-half of the affine strain energy in (1). The contribution of constraints to the free energy is given as

$$
W_{\mathrm{c}}=\frac{1}{2} n k \Theta \sum_{i}\left[B_{i}+D_{i}-\ln \left(B_{i}+1\right)-\ln \left(D_{i}+1\right)\right]
$$

for

$$
\begin{gathered}
B_{i}=\kappa^{2}\left(\lambda_{i}^{2}-1\right)\left(\lambda_{i}^{2}+\kappa\right)^{-2}, \\
D_{i}=\lambda_{i}^{2} \kappa^{-1} B_{i}
\end{gathered}
$$


and where $\kappa$ is a measure of the strengths of the constraints which depends on the relative sizes of free (phantom) fluctuations and actual constrained fluctuations. This model depends on the parameters $n, \phi$ and $\kappa$. In it $\kappa \rightarrow \infty$ for completely constrained junctions and $\kappa \rightarrow 0$ in the phantom chain limit, $n$ can take on any large, positive value and $\phi$ must be greater than two.

Results of simulations with the Flory and Erman model appear in Fig. A2.

The model results shown in Figs A1 and A2 reveal that of these existing models, only the Ogden model captures the state of deformation dependence of deformation. However, in order to capture this behavior, the Ogden model required six parameters. 